\title{
Thyroid Hormone Abnormalities in Hemodialyzed Patients: Low Triiodothyronine As Well As High Reverse Triiodothyronine Are Associated With Increased Mortality
}

\author{
J. HORÁČEK ${ }^{1 *}$, S. DUSILOVÁ SULKOVÁ ${ }^{2,3 *}$, M. KUBIŠOVÁ ${ }^{3}$, R. ŠAFRÁNEK $^{2,3}$, \\ E. MALÍŘOVÁ ${ }^{4}$, M. KALOUSOVÁ ${ }^{5}$, I. SVILIAS ${ }^{1}$, J. MALÝ $^{1}$, L. SOBOTKA ${ }^{3}$, P. Ž́ $^{1}$ \\ *These authors contributed equally to this work
}

${ }^{1}$ Department of Internal Medicine IV, Charles University in Prague, Faculty of Medicine and University Hospital Hradec Kralove, ${ }^{2}$ Hemodialysis Center, University Hospital Hradec Kralove, ${ }^{3}$ Department of Internal Medicine III, Charles University in Prague, Faculty of Medicine and University Hospital Hradec Kralove, ${ }^{4}$ Department of Nuclear Medicine, Charles University in Prague, Faculty of Medicine and University Hospital Hradec Kralove, ${ }^{5}$ Institute of Clinical Chemistry and Laboratory Diagnostics, First Faculty of Medicine and General University Hospital, Charles University in Prague, Czech Republic

Received January 13, 2012

Accepted June 15, 2012

On-line August 8, 2012

\section{Summary}

Numerous abnormalities of thyroid hormones in end-stage renal disease (ESRD) have been described. Our aim was to analyze the impact of these abnormalities on survival. In 167 hemodialyzed ESRD patients, TSH and thyroid hormone levels (T4, fT4, T3, fT3, rT3) were determined. The patients were then prospectively followed up for up to 5 years and the possible impact of any observed abnormalities on their mortality was studied. Only $16.8 \%$ patients had all six tests within the reference range. The pattern of nonthyroidal illness syndrome was found in $56.3 \%$. Low T3 was particularly common (44.3\%), and clearly associated with increased 6- and 12-month mortality and decreased overall survival (log rank test, $P=0.007$ ). Independent of T3 levels (Spearman correlation, NS), increased rT3 was more frequently observed $(9.9 \%)$ than expected from the literature, and was also related to increased mortality and decreased survival (log rank test, $P=0.021$ ). Increased rT3 may be more common in ESRD patients than previously described, and together with decreased T3 it may serve as an indicator of poor prognosis in subsequent months.

\section{Key words}

Thyroid • Triiodothyronine - Reverse triiodothyronine • Hemodialysis • Survival

\section{Corresponding author}

Jiri Horacek, Charles University in Prague, Faculty of Medicine and University Hospital Hradec Kralove, Department of Internal Medicine IV, Sokolska 581, 50005 Hradec Kralove, Czech Republic. Fax: +420 495832 011. E-mail: horacek9@gmail.com

\section{Introduction}

The high mortality of end-stage renal disease (ESRD) patients on maintenance hemodialysis is believed to be a function of complex metabolic and regulatory disorders (Go et al. 2004). In particular, numerous abnormalities in endocrine regulation have been described. Thyroid homeostatic regulation may be affected at several levels (Kaptein 1996, Lim 2001, Iglesias and Diez 2009). Hypothyroidism seems to be more common in chronic kidney disease patients (Lo et al. 2005), and its association with increased mortality has been reported (Enia et al. 2007). Moreover, patients with ESRD and hypothyroidism share some common symptomatology, e.g. asthenia, pallor and hypothermia; therefore correct interpretation of their thyroid status is essential, though this is complicated by both pathophysiological processes and methodological difficulties (Witzke et al. 2007). 
In particular, ESRD may be viewed as a severe nonthyroidal illness, leading to nonthyroidal illness syndrome (NTIS), with a decrease in triiodothyronine (T3), and in more severe cases also in thyroxine (T4) levels, not accompanied by the usual feed-back increase in thyroid-stimulating hormone (TSH) (Docter et al. 1993, Adler and Wartofsky 2007). However, it has been assumed that an increase in reverse triiodothyronine (rT3), a common abnormality in NTIS, does not occur in ESRD (Kaptein 1996, Iglesias and Diez 2009).

Hemodialysis strategy as well as dialysis demography has changed completely since 1980s (Lameire et al. 2009, Cavalli et al. 2010), when most of the descriptive studies on thyroid hormone patterns in ESRD were published (Lim et al. 1977, Faber et al. 1983, Kaptein et al. 1983a,b) and therefore some conclusions derived from those studies may not now be valid.

In several studies of ESRD, low T3 levels have been reported as an unfavourable prognostic factor for survival (Zoccali et al. 2006, Enia et al. 2007, Carrero et al. 2007); moreover, pretransplant low T3 levels may be associated with higher risk of kidney graft failure (Rotondi et al. 2008). A similar prognostic link with rT3 has not yet been published.

In this study we performed a cross-sectional survey of thyroid hormone levels in hemodialyzed ESRD patients, and we found numerous abnormalities, including high rT3. In addition, these patients were prospectively followed up for up to 5 years, and the possible impact of any observed abnormalities on their mortality was studied.

\section{Methods}

Patients with ESRD included in the chronic hemodialysis programme at our university hospital were sampled for thyroid function tests in 2006, 2008 and 2010. None of them had a history of thyroid disorders, and they were not treated with any drugs known to affect thyroid function. Patients were dialyzed three times weekly, using a two needle system, low-flux polysulphone dialyser $1.6-1.8 \mathrm{~m}^{2}$, bicarbonate dialysis solution with dialysate flow $500 \mathrm{ml} / \mathrm{min}$ and blood flow rate $300 \mathrm{ml} / \mathrm{min}$. Equilibrated $\mathrm{Kt} / \mathrm{V}$ was checked monthly and targeted above 1.2, in accordance with $\mathrm{K} / \mathrm{DOQI}$ guidelines (National Kidney Foundation 2006). The dialysis population studied was unselected but patients with severe acute complications requiring hospitalization at the time of sampling were not included in the analysis.
As the possible impact of thyroid hormone abnormalities on survival may be time-dependent (i.e. more significant in the shorter interval), only the last sample before the last follow up or death was used for calculations in those who were tested repeatedly. Altogether in 167 patients, (97 male, 70 female, median age 64.9 years) thyroid hormone levels were analyzed, and the patients were followed up for at least 540 days unless they died earlier. The study was in accordance with the Declaration of Helsinki (2000) of the World Medical Association, and was approved by the institutional ethics committee.

As heparin may interfere with competitive assays, blood was drawn just before the start of hemodialysis procedure from the inserted dialysis needle or from the arterial port of dialysis circuit, before contact of blood with dialyser and before heparin administration. This ensured that there was an interval of at least 48 hours since the last heparin application.

Thyroid function tests were performed in our radioisotope laboratory as described previously (Horacek et al. 2010); in addition, total T4, total T3 and reverse T3 were also assayed by radioimmunoassays (RIA). The battery of tests thus included: (a) TSH determined by immunoradiometric assay (IRMA, Immunotech, Beckman Coulter, Prague, Czech Republic), reference range (RR) 0.15-5.0 $\mathrm{mIU} / 1$, inter-assay variation (CV) $5.5 \%$; (b) FT4 measured by RIA (Immunotech, Beckman Coulter), RR 11.0-23.0 pmol/1, CV $8.4 \%$; (c) FT3, RIA (Immunotech, Beckman Coulter), RR 2.5-5.7 pmol/1, CV $6.4 \%$ ); (d) T4, RIA (Immunotech, Beckman Coulter), RR 70.0-140.0 nmol/l, CV 8.6\%); (e) T3, RIA (Immunotech, Beckman Coulter), RR 1.0-3.0 nmol/1, CV $7.7 \%$ ); and (f) rT3, RIA (Adaltis, Casalecchio di Reno, Italy), RR 0.14-0.54 nmol/1 CV $8.7 \%$ ).

The descriptive results were tentatively summarized in terms of proportions of various abnormalities, namely hypothyroidism, hyperthyroidism and NTIS, although the links between thyroid hormone levels and the typical patterns of these syndromes are less clear in chronic renal failure (Kaptein 1996), and subject to some debate. Spearman correlation was used for quantifying possible associations between the thyroid hormones. Patients surviving more than 6 and 12 months after the sampling were compared with those having died within the respective period using Mann-Whitney test, and for significantly different parameters (i.e. T3 and rT3), survival analysis using Kaplan-Meier curves and log rank test was performed. For statistical analysis, NCSS software package was used. 
Table 1. Thyroid function tests in 167 hemodialyzed ESRD patients (age: median 64.9 years, interquartile range (IQR) 56.2-75.7; Kt/V eq: median 1.31, IQR 1.11-1.47).

\begin{tabular}{llllll}
\hline \multirow{2}{*}{ Variable (unit) } & \multirow{2}{*}{$\begin{array}{l}\text { Reference } \\
\text { range (RR) }\end{array}$} & Median (IQR) & \multicolumn{3}{c}{ Percentage (\%) } \\
\cline { 5 - 6 } & & & normal & $<$ RR & $>$ RR \\
\hline$T S H(m I U / l)$ & $0.15-5.0$ & $1.82(1.16-3.32)$ & 85.0 & 3.6 & 11.4 \\
$T 4(\mathrm{nmol} / \mathrm{l})$ & $70-140$ & $71.4(60.9-84.4)$ & 53.9 & 45.5 & 0.6 \\
$T 3(\mathrm{nmol} / \mathrm{l})$ & $1.0-3.0$ & $1.06(0.83-1.26)$ & 55.7 & 44.3 & 0 \\
$r T 3(\mathrm{nmol} / \mathrm{l})$ & $0.14-0.54$ & $0.29(0.22-0.39)$ & 87.3 & 2.8 & 9.9 \\
$f T 4(\mathrm{pmol} / \mathrm{l})$ & $11.0-25.0$ & $11.6(10.1-13.5)$ & 61.7 & 38.3 & 0 \\
$f T 3(\mathrm{pmol} / \mathrm{l})$ & $2.5-5.8$ & $3.24(2.77-3.78)$ & 83.8 & 15.0 & 1.2 \\
\hline
\end{tabular}

\section{Results}

In our sample of 167 unselected ESRD patients, thyroid abnormalities were very common (Table 1), and only $16.8 \%$ patients had all the six tests within the reference ranges employed. The most common abnormalities were low $\mathrm{T} 4$ and low $\mathrm{T} 3$, occurring in $45.5 \%$ and $44.3 \%$ of cases, respectively.

Though rT3 was mostly normal, there were still $9.9 \%$ patients with levels above the reference range, considerably more than expected from the literature (Kaptein 1996, Witzke et al. 2007 while decreased levels were exceptional (2.8\%).

As the competitive assays for free hormones (fT3 and fT4) generally give less reliable results in CRF, due to methodological problems (Kaptein 1996), we put more emphasis on the more robust total hormone assays but even then there were only $28.7 \%$ patients with all the four tests (i.e. TSH, T4, T3 and rT3) within normal ranges. Patients with higher TSH and normal or lower T4 and $\mathrm{T} 3$ are normally classified as having subclinical or overt hypothyroidism, respectively; in our sample there were $19 / 167$ (i.e. $11.4 \%$ ) patients in this category. Similarly, patients with suppressed TSH and normal or higher T4 and T3 are usually classified as having subclinical or overt hyperthyroidism; in our sample they comprised 6/167, i.e. $3.6 \%$. The remaining 94/167 (56.3\%) patients were classified as NTIS; they had normal TSH levels together with (a) lower T3 and/or (b) lower T4 and/or (c) higher rT3.

The Spearman correlation analysis (Table 2) demonstrated some expected associations, e.g. significant inverse correlations between TSH and thyroid hormones, though they were rather weak ( $\rho$ coefficients ranging from -0.190 to -0.294 ) and did not include T3 $(\rho=-0.072$ ), probably reflecting the frequent incidence of
NTIS, or positive correlations between total and free hormones, again less prominent in T3/fT3 $(\rho=0.269)$ than in T4/fT4 $(\rho=0.575)$. Unexpectedly for such prevalent NTIS, no significant inverse correlation was found between T3 and rT3 $(\rho=-0.148$, NS). Conversely, an unexpectedly strong positive correlation between rT3 and both T4 $(\rho=0.448 ; P<0.001)$ and fT4 $(\rho=0.544 ; P<0.001)$ was revealed.

Table 2. Spearman correlation matrix of thyroid function tests in 167 hemodialyzed ESRD patients.

\begin{tabular}{rrrrrrr}
\hline & & fT4 & T4 & \multicolumn{1}{c}{ fT3 } & \multicolumn{1}{c}{ T3 } & \multicolumn{1}{c}{ rT3 } \\
\hline \multirow{2}{*}{$T S H$} & $\rho$ & -0.294 & -0.204 & -0.190 & -0.072 & -0.242 \\
& $P$ & $<0.001$ & 0.008 & 0.014 & 0.358 & 0.004 \\
$f T 4$ & $\rho$ & & 0.575 & 0.123 & 0.009 & 0.544 \\
& $P$ & & $<0.001$ & 0.114 & 0.904 & $<0.001$ \\
$T 4$ & $\rho$ & & & -0.092 & 0.344 & 0.448 \\
& $P$ & & & 0.237 & $<0.001$ & $<0.001$ \\
$f T 3$ & $\rho$ & & & & 0.269 & -0.109 \\
& $P$ & & & & $<0.001$ & 0.195 \\
$T 3$ & $\rho$ & & & & & -0.148 \\
& $P$ & & & & & 0.078 \\
& & & & & &
\end{tabular}

$\rho=$ Spearman rho correlation coefficient, $P=$ coefficient of significance

Within one year of sampling, 48 patients $(28.7 \%)$ died. Their T3 and rT3 levels were clearly different from those having survived longer than one year while there was no significant difference in any other hormone levels (Table 3). Similar findings were seen for the shorter 6 month period (Table 4), together suggesting an association of mortality at 6 to 12 months with lower T3 and higher rT3. 
Table 3. Comparison of hemodialyzed ESRD patients surviving one year since sampling with those dying within one year.

\begin{tabular}{llll}
\hline Variable (unit) & $\begin{array}{l}\text { Surviving one year } \\
\mathbf{n}=\mathbf{1 1 9}\end{array}$ & $\begin{array}{l}\text { Dying within one year } \\
\mathbf{n = 4 8}\end{array}$ & $\boldsymbol{P}$ \\
\hline TSH $(\mathrm{mlU} / \mathrm{l})$ & $1.80(1.20-3.38)$ & $2.03(1.10-3.00)$ & 0.992 \\
$T 4$ (nmoll) & $73.2(60.9-85.9)$ & $70.8(61.4-82.4)$ & 0.570 \\
$T 3(\mathrm{nmol} / \mathrm{l})$ & $1.08(0.89-1.27)$ & $0.95(0.73-1.19)$ & 0.030 \\
rT3 $(\mathrm{nmol} / \mathrm{l})$ & $0.26(0.21-0.35)$ & $0.36(0.29-0.48)$ & $<0.001$ \\
$f T 4(\mathrm{pmol} / \mathrm{l})$ & $11.8(10.3-13.5)$ & $11.1(9.5-13.6)$ & 0.299 \\
$f T 3(\mathrm{pmol} / \mathrm{l})$ & $3.27(2.83-3.78)$ & $3.19(2.65-3.78)$ & 0.366 \\
\hline
\end{tabular}

Values are expressed as median (interquartile range). Mann-Whitney test was used for comparison.

Table 4. Comparison of hemodialyzed ESRD patients surviving 6 months since sampling with those dying within 6 months.

\begin{tabular}{llll}
\hline Variable (unit) & $\begin{array}{l}\text { Surviving six months } \\
\mathbf{n = 1 4 2}\end{array}$ & $\begin{array}{l}\text { Dying within six months } \\
\mathbf{n = 2 5}\end{array}$ & $\boldsymbol{P}$ \\
\hline$T S H(\mathrm{mll} / \mathrm{l})$ & $1.94(1.20-3.40)$ & $1.55(0.89-2.57)$ & 0.258 \\
$T 4(\mathrm{nmol} / \mathrm{l})$ & $72.4(61.3-84.4)$ & $68.0(56.2-84.4)$ & 0.561 \\
$T 3(\mathrm{nmol} / \mathrm{l})$ & $1.09(0.88-1.27)$ & $0.80(0.71-1.06)$ & $<0.001$ \\
$r T 3(\mathrm{nmol} / \mathrm{l})$ & $0.28(0.22-0.38)$ & $0.39(0.31-0.47)$ & 0.015 \\
$f T 4(\mathrm{pmol} / \mathrm{l})$ & $11.7(10.2-13.5)$ & $11.4(9.9-14.8)$ & 0.866 \\
$f T 3(\mathrm{pmol} / \mathrm{l})$ & $3.23(2.80-3.74)$ & $3.50(2.66-3.88)$ & 0.986 \\
\hline
\end{tabular}

Values are expressed as median (interquartile range). Mann-Whitney test was used for comparison.

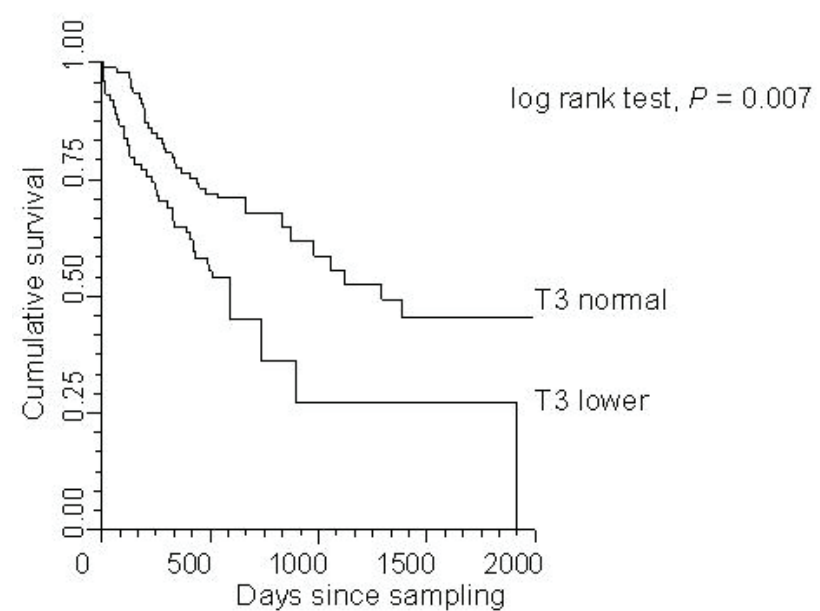

Fig. 1. Kaplan-Meier survival analysis of T3 in ESRD patients. Patients are divided into two groups on the basis of their T3 level; upper trace: values within the reference range (1.0-3.0 $\mathrm{nmol} / \mathrm{l})$; lower trace: values $<1.0 \mathrm{nmol} / \mathrm{l}$. Log rank test, $P=0.007$.

These associations were confirmed by survival analysis. The patients with T3 below RR $(<1 \mathrm{nmol} / \mathrm{l})$ had significantly worse survival than those within it (log rank test, $P=0.007$; Kaplan-Meier, Figure 1). When median

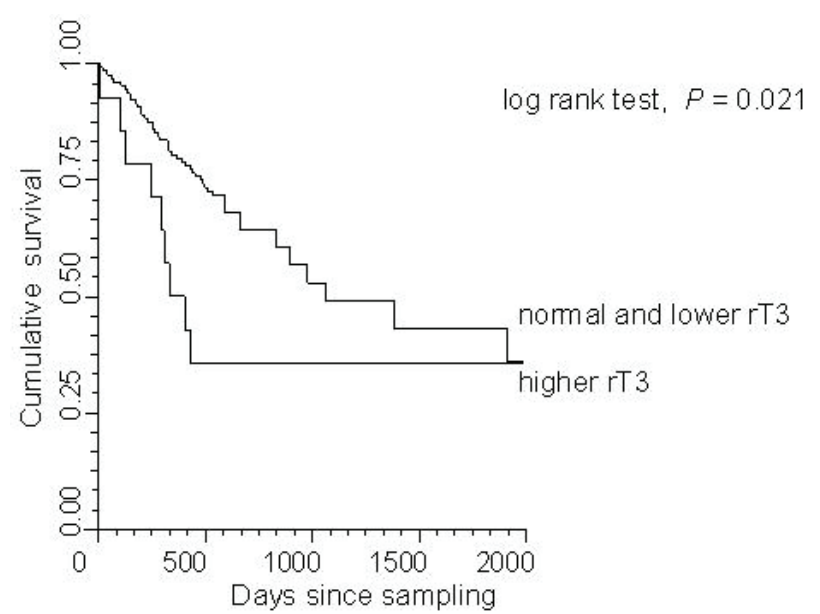

Fig. 2. Kaplan-Meier survival analysis of $r T 3$ in ESRD patients. Patients are divided into two groups on the basis of their rT3 level; upper trace: values within the reference range (0.14-0.54 $\mathrm{nmol} / \mathrm{l}$ ) or below; lower trace: values $>0.54 \mathrm{nmol} / \mathrm{l}$. Log rank test, $P=0.021$.

was used as the discriminator then patients with T3 values below median $(<1.06 \mathrm{nmol} / \mathrm{l})$ had worse survival than those above median (log rank test, $P=0.042$ ). Similarly for rT3, the patients with values above RR 
$(>0.54 \mathrm{nmol} / \mathrm{l})$ had significantly worse survival than the rest of the sample (both within and below RR; log rank test, $P=0.021$; Kaplan-Meier, Figure 2). Also, those above median $(>0.29 \mathrm{nmol} / \mathrm{l})$ had worse survival than those below it (log rank test, $P=0.020)$.

\section{Discussion}

Normal thyroid status was found only in a minority of our ESRD patients, and more than a half of our sample fulfilled the criteria for NTIS. In addition, we have used TSH values out of the reference range as markers of hypo/hyperthyroidism in the traditional way (Jameson and Weetman 2008), and in accord with the classification used in population studies (Lo et al. 2005, Hollowell et al. 2002) though in chronic renal failure a minor increase or decrease in TSH may also be attributed to NTIS (Kaptein 1996). If some of those classified as hypo/hyperthyroid actually had NTIS instead then the proportion of patients with the syndrome would have even been higher.

Interestingly, no significant difference in survival was found between the whole group of NTIS patients and those with normal values of TSH, T4, T3 and rT3 (data not shown). When analyzed separately, low T3 as well as high rT3 were both associated with decreased survival, especially within the first year of follow-up. This suggests that low T4/normal TSH pattern, though often considered as severe NTIS (Adler and Wartofsky 2007), may be of less prognostic importance in hemodialyzed patients.

Several authors have recently pointed out the negative prognostic value of low T3 in ESRD (Zoccali et al. 2006, Enia et al. 2007, Carrero et al. 2007). In our hemodialyzed ESRD patients, we confirmed these conclusions. Clearly, when low T3 is found in an ESRD patient it may serve as a warning sign of poor prognosis within the coming months. It suggests that identifying and treating any modifiable abnormalities that may cause both low T3 and increased mortality (e.g. malnutrition, inflammation, etc.) can be of benefit to the patient. Conversely, misguided attempts to replete thyroid hormone stores by exogenous supplementation may worsen the prognosis (Lim 2001).

Data on rT3 levels in hemodialyzed patients are so far scarce. The representative reviews (Kaptein 1996, Iglesias and Diez 2009) state that patients with ESRD have normal total serum rT3 rather than the elevated levels observed in NTIS, referring to earlier studies from 1983 (Faber et al. 1983, Kaptein et al. 1983a,b). Though these studies give careful and detailed description of thyroid hormone kinetics in a small sample of ESRD patients (ranging from 6 to 13, of which only 5 were chronically hemodialyzed) they do not provide relevant data on the estimated proportions of rT3 abnormalities in ESRD population. Such an estimate may be derived from a more recent paper (Witzke et al. 2007) comparing thyroid hormones, including rT3, in young patients with preterminal $(n=48)$ and terminal $(n=288)$ renal failure. Their hemodialyzed ESRD patients had significantly lower rT3 $(0.25 \pm 0.02 \mathrm{nmol} / \mathrm{l}$; mean \pm SEM $)$ than those with preterminal renal failure $(0.39 \pm 0.03 \mathrm{nmol} / \mathrm{l})$ or healthy controls $(0.37 \pm 0.02 \mathrm{nmol} / \mathrm{l})$. As they also used RIA with the same RR for the rT3 assay our results should be comparable with theirs. However, when expressed in the same way, the rT3 levels in our ESRD patients were $0.36 \pm 0.03 \mathrm{nmol} / \mathrm{l}$, i.e. clearly higher than in their ESRD group, and similar to their other groups.

Most of our ESRD patients (ca. $87 \%$ ) had rT3 within the RR, in accord with the prevailing opinion (Kaptein 1996, Iglesias and Diez 2009). However, and rather unexpectedly, there were ca. $10 \%$ patients with an increased rT3 in our sample. Moreover, the higher rT3 was clearly associated with poor prognosis. These novel findings suggest that also in ESRD (as a special case of NTIS) rT3 may be elevated, and serve as a negative prognostic marker, similar to other severe clinical conditions (Peeters et al. 2005).

The observed negative prognostic value of high rT3 appeared independent of low T3 since there was no significant (inverse) correlation between the variables. Conversely, a moderately tight and highly significant positive correlation was found between rT3 and both T4 and fT4. Though this would suggest parallel variations in 5 '-deiodinase activity no ready explanation is currently available. If type 2 deiodinase activity fluctuations were responsible, one would expect an inverse correlation between T3 and rT3; perhaps both type 1 and type 2 deiodinases are involved (Maia et al. 2011, Williams and Bassett 2011). Interestingly, a similar correlation between rT3 and fT4 was found in independently living elderly men, and higher rT3 and fT4 were associated with lower levels of physical function (van den Beld et al. 2005), also suggestive of worse prognosis.

\section{Conclusion}

Low T3 concentrations have recently been associated with poor prognosis in ESRD. Our results are 
in accordance with these conclusions. Moreover, in this study we focused on a detailed description of thyroid status in a large cohort of non-selected ESRD patients and we reveal two novel findings concerning rT3: levels are higher than previously described and higher rT3 is associated with worse prognosis. These results are worthy of further investigation.

\section{Conflict of Interest}

There is no conflict of interest.

\section{Acknowledgements}

The work was supported by the research project MZO 0021620819 and by the grant SVV-2012-264903. The authors wish to thank Ing. Josef Bukac, MS, PhD (Department of Biophysics, Faculty of Medicine in Hradec Kralove) for the survival analysis and statistical consultations and Catherine Mc Grath, MD (Queen Elizabeth Hospital, Birmingham, UK) for linguistic review of the manuscript.

\section{References}

ADLER SM, WARTOFSKY L: The nonthyroidal illness syndrome. Endocrinol Metab Clin North Am 36: 657-672, 2007.

CARRERO JJ, QURESHI AR, AXELSSON J, YILMAZ MI, REHNMARK S, WITT MR, BÁRÁNY P, HEIMBÜRGER O, SULIMAN ME, ALVESTRAND A, LINDHOLM B, STENVINKEL P: Clinical and biochemical implications of low thyroid hormone levels (total and free forms) in euthyroid patients with chronic kidney disease. J Intern Med 262: 690-701, 2007.

CAVAlli A, Del VECCHIO L, MANZONI C, LOCATELli F: Hemodialysis: yesterday, today and tomorrow. Minerva Urol Nefrol 62: 1-11, 2010.

DOCTER R, KRENNING EP, DE JONG M, HENNEMANN G: The sick euthyroid syndrome: changes in thyroid hormone serum parameters and hormone metabolism. Clin Endocrinol 39: 499-518, 1993.

ENIA G, PANUCCIO V, CUTRUPI S, PIZZINI P, TRIPEPI G, MALLAMACI F, ZOCCALI C: Subclinical hypothyroidism is linked to micro-inflammation and predicts death in continuous ambulatory peritoneal dialysis. Nephrol Dial Transplant 22: 538-544, 2007.

FABER J, HEAF J, KIRKEGAARD C, LUMHOLTZ IB, SIERSBAEK-NIELSEN K, KØLENDORF K, FRIIS T: Simultaneous turnover studies of thyroxine, 3,5,3' and 3,3',5'-triiodothyronine, 3,5-, 3,3', and 3'5'diiodothyronine, and 3'-monoiodothyronine in chronic renal failure. J Clin Endocrinol Metab 56: 211-217, 1983.

GO AS, CHERTOW GM, FAN D, MCCULLOCH CE, HSU CY: Chronic kidney disease and the risk of death, cardiovascular events and hospitalization. $N$ Engl J Med 351: 1296-1305, 2004.

HOLLOWELL JG, STAEHLING NW, FLANDERS WD, HANNON WH, GUNTER EW, SPENCER CA, BRAVERMAN LE: Serum TSH, T4, and thyroid antibodies in the United States population (1988 to 1994): National Health and Nutrition Examination Survey (NHANES III). J Clin Endocrinol Metab 87: 489-499, 2002.

HORACEK J, SPITALNIKOVA S, DLABALOVA B, MALIROVA E, VIZDA J, SVILIAS I, CEPKOVA J, MC GRATH C, MALY J: Universal screening detects two-times more thyroid disorders in early pregnancy than targeted high-risk case finding. Eur J Endocrinol 163: 645-650, 2010.

IGLESIAS P, DIEZ JJ: Thyroid dysfunction and kidney disease. Eur J Endocrinol 160: 503-515, 2009.

JAMESON JL, WEETMAN AP: Disorders of the thyroid gland. In: Harrison's Principles of Internal Medicine (1 ${ }^{\text {th }}$ ed.). AS FAUCI, E BRAUNWALD, DL KASPER, SL HAUSER, DL LONGO, JL JAMESON, J LOSCALZO (eds), McGraw-Hill Companies Inc, New York, 2008, pp 2224-2247.

KAPTEIN EM: Thyroid hormone metabolism and thyroid diseases in chronic renal failure. Endocrine Rev 17: 45-63, 1996.

KAPTEIN EM, FEINSTEIN EI, NICOLOFF JT, MASSRY GD: Serum reverse triiodothyronine and thyroxine kinetics in patients with chronic renal failure. J Clin Endocrinol Metab 57: 181-189, 1983a. 
KAPTEIN EM, FEINSTEIN EI, NICOLOFF JT, MASSRY GD: Alterations of serum reverse triiodothyronine and thyroxine kinetics in chronic renal failure: Role of nutritional status, chronic illness, uremia, and hemodialysis. Kidney Int 24 (Suppl 16): S180-S186, 1983b.

LAMEIRE N, VAN BIESEN W, VANHOLDER R: Did 20 years of technological innovations in hemodialysis contribute to better patient outcomes? Clin J Am Soc Nephrol 4 (Suppl 1): S30-S40, 2009.

LIM VS: Thyroid function in patients with chronic renal failure. Am J Kidney Dis 38: S80-S84, 2001.

LIM VS, FANG VS, KATZ AI, REFETOFF S: Thyroid dysfunction in chronic renal failure. J Clin Invest 60: 522-534, 1977.

LO JC, CHERTOW GM, GO AS, HSU CY: Increased prevalence of subclinical and clinical hypothyroidism in persons with chronic kidney disease. Kidney Int 67: 1047-1052, 2005.

MAIA AL, GOEMANN IM, MEYER ELS, WAJNER SM: Type 1 iodothyronine deiodinase in human physiology and disease. J Endocrinol 209: 283-297, 2011.

NATIONAL KIDNEY FOUNDATION, HEMODIALYSIS ADEQUACY 2006 WORK GROUP: Clinical practice guidelines for hemodialysis adequacy, update 2006. Am J Kidney Dis 48 (Suppl 1): S2-S90, 2006.

PEETERS RP, WOUTERS PJ, VAN TOOR H, KAPTEIN E, VISSER TJ, VAN DEN BERGHE G: Serum 3,3',5'triiodothyronine $\left(\mathrm{rT}_{3}\right)$ and 3,5,3'-triiodothyronine/rT $\mathrm{r}_{3}$ are prognostic markers in critically ill patients and are associated with postmortem tissue deiodinase activities. J Clin Endocrinol Metab 90: 4559-4565, 2005.

ROTONDI M, NETTI GS, ROSATI A, MAZZINGHI B, MAGRI F, RONCONI E, BECHERUCCI F, PRADELLA F, SALVADORI M, SERIO M, ROMAGNANI P, CHIOVATO L: Pretransplant serum FT3 levels in kidney graft recipients are useful for identifying patients with higher risk for graft failure. Clin Endocrinol 68: 220$225,2008$.

VAN DEN BELD AW, VISSER TJ, FEELDERS RA, GROBBEE DE, LAMBERTS SWJ: Thyroid hormone concentrations, disease, physical function, and mortality in elderly men. J Clin Endocrinol Metab 90: 64036409, 2005.

WILLIAMS GR, BASSETT JHD: Local control of thyroid hormone action: role of type 2 deiodinase. J Endocrinol 209: 261-272, 2011.

WITZKE O, WIEMANN J, PATSCHAN D, WU K, PHILIPP T, SALLER B, MANN K, REINHARDT W: Differential T4 degradation pathways in young patients with preterminal and terminal renal failure. Horm Metab Res 39: 355-358, 2007.

ZOCCALI C, MALLAMACI F, TRIPEPI G, CUTRUPI S, PIZZINI P: Low triiodothyronine and survival in end-stage renal disease. Kidney Int 70: 523-528, 2006. 